12th International Symposium on Cosmology and

Particle Astrophysics (CosPA 2015)

International Journal of Modern Physics: Conference Series

Vol. 43 (2016) 1660201 (9 pages)

(C) The Author(s)

DOI: $10.1142 / \mathrm{S} 2010194516602015$

\title{
Gravitational Compton Scattering from the Worldline Formalism
}

\author{
Naser Ahmadiniaz \\ Center for Relativistic Laser Science, Institute for Basic Science, 61005 Gwangju, Korea \\ ahmadiniaz@ibs.re.kr \\ Olindo Corradini \\ Dipartimento di Scienze Fisiche, Informatiche e Matematiche, \\ Università degli Studi di Modena e Reggio Emilia, Via Campi 213/A, I-41125 Modena, Italy, \\ and INFN, Sezione di Bologna, Via Irnerio 46, I-40126 Bologna, Italy \\ olindo.corradini@unimore.it \\ José Manuel Dávila \\ Facultad de Ciencias, Universidad Autónoma del Estado de México, Instituto Literario 100, \\ C.P. 50000, Toluca, México. \\ tbcsdu@yahoo.com \\ Christian Schubert \\ Instituto de Física y Matemáticas Universidad Michoacana de San Nicolás de Hidalgo Edificio \\ C-3, Apdo. Postal 2-82 C.P. 58040, Morelia, Michoacán, México \\ schubert@ifm.umich.mx \\ Published 7 July 2016
}

\begin{abstract}
We report on an ongoing study of photon amplitudes, graviton amplitudes and mixed photon-graviton amplitudes at tree-level using the worldline formalism. We explicitly recalculate the amplitude with one photon and one graviton coupled to a scalar propagator, relevant for graviton photoproduction. We comment on the factorization properties of this amplitude, and outline a generalization to similar processes involving more gravitons.
\end{abstract}

Keywords: Photon-graviton; Compton scattering; worldline formalism.

PACS numbers: 04.60.-m, 04.62.+v, 14.70.Bh, 14.70.Kv

\section{Motivation}

As shown in Refs. 1, 2, 3 the elastic scattering of gravitons from an elementary particle with arbitrary spin can be factorized. The presence of gravitons in these

This is an Open Access article published by World Scientific Publishing Company. It is distributed under the terms of the Creative Commons Attribution 4.0 (CC-BY) License. Further distribution of this work is permitted, provided the original work is properly cited. 


\section{N. Ahmadiniaz et al.}

elastic scatterings increases the number of Feynman diagrams which one has to calculate. For example, for the gravitational Compton scattering

$$
\gamma+s \rightarrow g+s
$$

where $s, \gamma$ and $g$ represent the scalar particle, photon and graviton, respectivelybesides the Born and seagull diagrams, one should include the contribution of the ' $\gamma$-pole' diagram ${ }^{2}$ which involves a triple coupling between one graviton and two photons. The inclusion of the latter diagram is necessary for maintaining general covariance. The same type of diagrams enter in the scattering with two gravitons, i.e. $g+s \rightarrow g+s$, where one needs an extra $g$-pole diagram which involves a triplegraviton coupling. Recently, some of the present authors have presented master formulas for a scalar propagator coupled to an abelian (photon) ${ }^{4}$ and non-abelian (gluon $)^{5}$ background using the worldline formalism. These master formulas are compact and could give some information about the non-perturbative regime of the corresponding theories. The main motivation of this line of work is to obtain a master formula for the mixed electromagnetic-gravitational as well as pure gravitational backgrounds, in other words a scalar particle interacting with any number of photons and gravitons. In the present manuscript we describe the first step, i.e. the worldline computation of the photon-graviton Compton scattering amplitude.

\section{The Worldline Formalism in Flat and Curved Spacetime}

The worldline formalism is an approach to amplitude calculations in quantum field theory based on first quantization. It has been introduced by Feynman ${ }^{6}$ in 1950 for scalar and in 1951 for spinor quantum electrodynamics (QED) (for a review see Ref. 7). Let us consider a scalar particle of mass $m_{i}$ propagating from point $x^{\prime}$ to point $x$ during the proper-time $T$ while interacting with an external abelian field $A$. In Feynman's representation, this propagator is written as

$$
\begin{aligned}
\Gamma\left[x, x^{\prime}\right] & =\int_{0}^{\infty} d T \mathrm{e}^{-m^{2} T} \int_{x(0)=x^{\prime}}^{x(T)=x} \mathcal{D} x(\tau) \mathrm{e}^{-S_{0}-S_{e}}, \\
S_{0} & =\int_{0}^{T} d \tau \frac{1}{4} \dot{x}^{2}, \\
S_{e} & =i e \int_{0}^{T} d \tau \dot{x}(\tau) \cdot A(x(\tau)) .
\end{aligned}
$$

Here, $S_{0}$ describes the free propagation and $S_{e}$ the interaction of the scalar particle with the background field. The simplest generalization of this worldline path integral to a curved background, i.e, to include gravity, is to introduce the background metric $g_{\mu \nu}(x(\tau))$. The free action then generalizes to

$$
S_{0}=\frac{1}{4} \int_{0}^{T} d \tau \dot{x}^{\mu} g_{\mu \nu}(x(\tau)) \dot{x}^{\nu} .
$$


In the context of graviton scattering, the metric can be considered as the flat metric plus some fluctuation term which is a function of space-time,

$$
g_{\mu \nu}(x(\tau))=\eta_{\mu \nu}+\kappa h_{\mu \nu}(x(\tau)) .
$$

This world line action for curved space-time leads to considerable mathematical subtleties, as have been discussed extensively in Ref. 8. We will not go into this in this short report, but just mention that general covariance leads one to redefine the naive integrand measure to a new one

$$
\begin{aligned}
\mathcal{D} x(\tau) & \rightarrow D x(\tau) \prod_{0 \leq \tau \leq T} \sqrt{\operatorname{det} g_{\mu \nu}(x(\tau))} \\
& =D x \int_{\mathrm{PBC}} D a D b D c \mathrm{e}^{-\int_{0}^{T} d \tau \frac{1}{4} g_{\mu \nu}\left[a^{\mu}(\tau) a^{\nu}(\tau)+b^{\mu}(\tau) c^{\nu}(\tau)\right]} .
\end{aligned}
$$

Here in the last equality we have introduced some extra ghost fields, with periodic boundary condition ('PBC'), to exponentiate the extra factor (see, e.g. Ref. 9). Thus the generalization of Eq. (1) to curved spacetime becomes

$$
\begin{aligned}
\Gamma\left[x, x^{\prime}\right]= & \int_{0}^{\infty} d T \mathrm{e}^{-m^{2} T} \int_{x(0)=x^{\prime}}^{x(T)=x} D x(\tau) \mathrm{e}^{-\int_{0}^{T} d \tau \frac{1}{4}\left(\dot{x}^{2}+a^{2}+b \cdot c\right)} \\
& \times \mathrm{e}^{-i e \int_{0}^{T} d \tau \dot{x} \cdot A(x)-\frac{1}{4} \int_{0}^{T} d \tau_{0} h_{\mu \nu}\left[\dot{x}^{\mu}\left(\tau_{0}\right) \dot{x}^{\nu}\left(\tau_{0}\right)+a^{\mu}\left(\tau_{0}\right) a^{\nu}\left(\tau_{0}\right)+b^{\mu}\left(\tau_{0}\right) c^{\nu}\left(\tau_{0}\right)\right]} .
\end{aligned}
$$

By choosing $A_{\mu}(x)=\sum_{l} \varepsilon_{l \mu} \mathrm{e}^{i k_{l} \cdot x\left(\tau_{l}\right)}$ and $h_{\mu \nu}(x)=\sum_{l} \epsilon_{l \mu \nu} \mathrm{e}^{i k_{l} \cdot x\left(\tau_{l}\right)}$ (plane-wave), we can define vertex operators for the photon and graviton coupled to the scalar propagator,

$$
\begin{aligned}
V^{\gamma}[\varepsilon, k] & =\varepsilon_{\mu} \int_{0}^{T} d \tau \dot{x}^{\mu} \mathrm{e}^{i k \cdot x}, \\
V^{h}\left[\epsilon, k_{0}\right] & =\epsilon_{\mu \nu} \int_{0}^{T} d \tau_{0}\left[\dot{x}_{0}^{\mu} \dot{x}_{0}^{\nu}+a_{0}^{\mu} a_{0}^{\nu}+b_{0}^{\mu} c_{0}^{\nu}\right] \mathrm{e}^{i k_{0} \cdot x\left(\tau_{0}\right)},
\end{aligned}
$$

where $\epsilon_{\mu \nu}$ is the symmetric graviton polarization tensor. The path integral is computed by splitting $x^{\mu}(\tau)$ into a background part $x_{\mathrm{bg}}^{\mu}$ which encodes the boundary conditions, and the fluctuation $q^{\mu}(\tau)$ which satisfies Dirichelet boundary conditions,

$$
\begin{aligned}
x(\tau) & =x_{\mathrm{bg}}(\tau)+q(\tau), \\
x_{\mathrm{bg}}(\tau) & =\frac{\left(x-x^{\prime}\right) \tau}{T}+x^{\prime}, \\
q(0) & =q(T)=0 .
\end{aligned}
$$

The propagator for the fluctuation part is given by ${ }^{8}$

$$
\left\langle q^{\mu}\left(\tau_{1}\right) q^{\nu}\left(\tau_{2}\right)\right\rangle=-2 \delta^{\mu \nu} \Delta\left(\tau_{1}, \tau_{2}\right)
$$




\section{N. Ahmadiniaz et al.}

with the worldline Green's function $\Delta\left(\tau_{i}, \tau_{j}\right)$,

$$
\Delta\left(\tau_{i}, \tau_{j}\right)=\frac{\tau_{i} \tau_{j}}{T}+\frac{\left|\tau_{i}-\tau_{j}\right|}{2}-\frac{\tau_{i}+\tau_{j}}{2} .
$$

We note that this Green function has a nontrivial coincidence limit

$$
\Delta(\tau, \tau)=\frac{\tau^{2}}{T}-\tau
$$

We will also need derivatives of (9), which read,

$$
\begin{aligned}
\Delta\left(\tau_{i}, \tau_{j}\right) & =\frac{\tau_{j}}{T}+\frac{1}{2} \operatorname{sign}\left(\tau_{i}-\tau_{j}\right)-\frac{1}{2}, \\
\Delta^{\bullet}\left(\tau_{i}, \tau_{j}\right) & =\frac{\tau_{i}}{T}-\frac{1}{2} \operatorname{sign}\left(\tau_{i}-\tau_{j}\right)-\frac{1}{2}, \\
\bullet \Delta^{\bullet}\left(\tau_{i}, \tau_{j}\right) & =\frac{1}{T}-\delta\left(\tau_{i}-\tau_{j}\right) .
\end{aligned}
$$

Here, we follow the notation of Ref. 8, where left and right dots indicate derivatives with respect to the first and second argument, respectively. The correlators for the ghost fields are simply

$$
\begin{gathered}
\left\langle a^{\mu}\left(\tau_{1}\right) a^{\nu}\left(\tau_{2}\right)\right\rangle=+2 \delta^{\mu \nu} \delta\left(\tau_{1}-\tau_{2}\right), \\
\left\langle b^{\mu}\left(\tau_{1}\right) c^{\nu}\left(\tau_{2}\right)\right\rangle=-4 \delta^{\mu \nu} \delta\left(\tau_{1}-\tau_{2}\right) .
\end{gathered}
$$

In the next section we use the vertex operators and propagators to calculate the tree-level amplitude with one photon and one graviton using the method developed in this section. Let us mention that, at the one-loop level, there have already been several applications of this curved-space world line formalism, for example, the lowenergy limit of photon-graviton amplitudes, ${ }^{10}$ gravitational corrections to the EulerHeisenberg Lagrangian, ${ }^{11}$ and photon-graviton conversion in an electromagnetic field. ${ }^{12}$

\section{Compton Scattering with One Graviton and One Photon}

In this section, we will analyze in detail the case of one graviton and one photon, both on-shell. Choosing $A(x)$ and $h_{\mu \nu}(x)$ as plane waves, and expanding the interaction exponentials to linear order, we have, in $x$-space,

$$
\begin{aligned}
\Gamma\left[x, x^{\prime}\right]= & (-i e)\left(-\frac{\kappa}{4}\right) \int_{0}^{\infty} d T \mathrm{e}^{-m^{2} T} \\
& \times \int_{x(0)=x^{\prime}}^{x(T)=x} D x(\tau) \int_{\mathrm{PBC}} D a D b D c \mathrm{e}^{-\frac{1}{4} \int_{0}^{T} d \tau\left(\dot{x}^{2}+a^{2}+b \cdot c\right)} V^{\gamma}[\varepsilon, k] V^{h}\left[\epsilon, k_{0}\right] .
\end{aligned}
$$

This path integral represents the first three diagrams in Fig. 1, i.e. the two 'Born diagrams' and the 'seagull diagram'. The fourth diagram, called ' $\gamma$-pole diagram', is not included in Eq. (13), and will be discussed later in this section. 

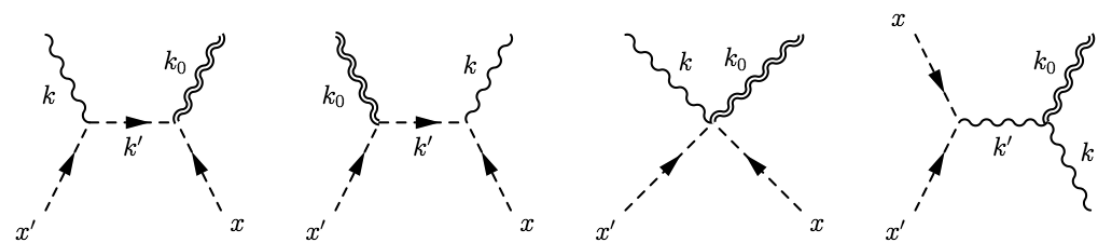

Fig. 1. Diagrams relevant to graviton photoproduction. From left to right we have two Born, one seagull and one $\gamma$-pole diagrams in momentum space.

After substituting Eq. (7) into Eq. (13) we get

$$
\begin{aligned}
\Gamma\left[x, x^{\prime}\right]= & i \frac{\kappa e}{4} \varepsilon_{\alpha} \epsilon_{\mu \nu} \int_{0}^{\infty} d T e^{-\frac{1}{4 T}\left(x-x^{\prime}\right)^{2}-m^{2} T} \int_{q(0)=0}^{q(T)=0} D q(\tau) \\
& \times \int_{\mathrm{PBC}} D a D b D c \mathrm{e}^{-\frac{1}{4} \int_{0}^{T} d \tau\left(\dot{q}^{2}+a^{2}+b \cdot c\right)} \\
& \times \int_{0}^{T} d \tau \int_{0}^{T} d \tau_{0} \mathrm{e}^{i\left(k \cdot x_{\mathrm{bg}}(\tau)+k_{0} \cdot x_{\mathrm{bg}}\left(\tau_{0}\right)\right)}\left(\frac{x-x^{\prime}}{T}+\dot{q}(\tau)\right)^{\alpha} \mathrm{e}^{i\left(k \cdot q(\tau)+k_{0} \cdot q\left(\tau_{0}\right)\right)} \\
& \times\left\{\left(\frac{x-x^{\prime}}{T}+\dot{q}\left(\tau_{0}\right)\right)^{\mu}\left(\frac{x-x^{\prime}}{T}+\dot{q}\left(\tau_{0}\right)\right)^{\nu}+a^{\mu}\left(\tau_{0}\right) a^{\nu}\left(\tau_{0}\right)+b^{\mu}\left(\tau_{0}\right) c^{\nu}\left(\tau_{0}\right)\right\} .
\end{aligned}
$$

The $q$ - path integral as well as the ghost path integrals are now gaussian, so that their evaluation boils down to Wick-contraction combinatorics. Using the propagators in Eq. (9) and Eq. (12), we arrive at

$$
\begin{aligned}
\Gamma\left[x^{\prime}, x_{-}\right]= & i \frac{\kappa e}{4} \mathrm{e}^{i\left(k+k_{0}\right) \cdot x^{\prime}} \int_{0}^{\infty} d T \mathrm{e}^{-\frac{x^{2}}{4 T}-m^{2} T}(4 \pi T)^{-\frac{D}{2}} \int_{0}^{T} d \tau d \tau_{0}\left\{\frac{\left(x_{-} \cdot \varepsilon\right)\left(x_{-} \epsilon x_{-}\right)}{T^{3}}\right. \\
& -\frac{4 i}{T^{2}}\left(\varepsilon \cdot x_{-}\right)\left(k \epsilon x_{-}\right)^{\bullet} \Delta\left(\tau_{0}, \tau\right)-\frac{4}{T}\left(\varepsilon \cdot x_{-}\right)(k \epsilon k)^{\bullet} \Delta^{2}\left(\tau_{0}, \tau\right) \\
& -\frac{2 i}{T^{2}}\left(\varepsilon \cdot k_{0}\right)\left(x_{-} \epsilon x_{-}\right) \Delta^{\bullet}\left(\tau_{0}, \tau\right)-\frac{4}{T}\left(\varepsilon \epsilon x_{-}\right)^{\bullet} \Delta^{\bullet}\left(\tau_{0}, \tau\right) \\
& -\frac{8}{T}\left(\varepsilon \cdot k_{0}\right)\left(k \epsilon x_{-}\right)^{\bullet} \Delta\left(\tau_{0}, \tau\right) \Delta^{\bullet}\left(\tau_{0}, \tau\right)+8 i(k \epsilon \varepsilon)^{\bullet} \Delta^{\bullet}\left(\tau_{0}, \tau\right) \bullet \Delta\left(\tau_{0}, \tau\right) \\
& \left.+8 i(k \epsilon k)\left(k_{0} \cdot \varepsilon\right)^{\bullet} \Delta\left(\tau_{0}, \tau\right)^{2} \Delta^{\bullet}\left(\tau_{0}, \tau\right)\right\} \mathrm{e}^{2 k_{0} \cdot k \Delta\left(\tau_{0}, \tau\right)+\frac{i}{T}\left(k \tau+k_{0} \tau_{0}\right) \cdot x_{-}}
\end{aligned}
$$

where the new variables are defined as

$$
x_{-}=x-x^{\prime}, \quad 2 x_{+}=x+x^{\prime} .
$$




\section{N. Ahmadiniaz et al.}

Now we Fourier transform to momentum space:

$$
\begin{aligned}
\Gamma\left[p, p^{\prime}\right] & =\int d^{D} x^{\prime} d^{D} x \mathrm{e}^{i p^{\prime} \cdot x^{\prime}+i p \cdot x} \Gamma\left[x, x^{\prime}\right] \\
& =\int d^{D} x_{+} d^{D} x_{-} \mathrm{e}^{i x_{+} \cdot\left(p^{\prime}+p\right)+i x_{-} \cdot\left(\frac{p}{2}-\frac{p^{\prime}}{2}\right)} \Gamma\left[x_{+}, x_{-}\right] .
\end{aligned}
$$

After rewriting Eq. (15) in terms of the new variables and plugging into Eq. (17), the $x_{+}$integral just produces the delta function for energy-momentum conservation, which we omit in the following. The $x_{-}$integral is gaussian and can be evaluated easily. Rescaling the proper-time as $\tau=T u$, and redefining the worldline Green's function as $\Delta\left(u, u^{\prime}\right)=u u^{\prime}+\frac{\left|u-u^{\prime}\right|}{2}-\frac{u+u^{\prime}}{2}$, the result can be written as

$$
\begin{aligned}
\Gamma\left[p, p^{\prime}\right]= & i \frac{\kappa e}{4} \int_{0}^{\infty} d T \int_{0}^{1} d u d u_{0}\left\{8 i\left[T(\varepsilon \epsilon l)-T^{2}(\varepsilon \cdot l)(l \epsilon l)\right]\right. \\
& +4 i\left[4 T^{2}(\varepsilon \cdot l)(k \epsilon l)-2 T(\varepsilon \epsilon k)\right] \bullet \Delta\left(u_{0}, u\right)-8 i T^{2}(\varepsilon \cdot l)(k \epsilon k)^{\bullet} \Delta^{2}\left(u_{0}, u\right) \\
& +8 i T^{2}\left(\varepsilon \cdot k_{0}\right)(l \epsilon l) \Delta^{\bullet}\left(u_{0}, u\right)-8 i T(\varepsilon \epsilon l) \bullet \Delta^{\bullet}\left(u_{0}, u\right) \\
& -16 i T^{2}\left(\varepsilon \cdot k_{0}\right)(k \epsilon l)^{\bullet} \Delta\left(u_{0}, u\right) \Delta^{\bullet}\left(u_{0}, u\right)+8 i T(\varepsilon \epsilon k) \bullet \Delta^{\bullet}\left(u_{0}, u\right)^{\bullet} \Delta\left(u_{0}, u\right) \\
& \left.+8 i T^{2}\left(\varepsilon \cdot k_{0}\right)(k \epsilon k)^{\bullet} \Delta\left(u_{0}, u\right)^{2} \Delta^{\bullet}\left(u_{0}, u\right)\right\} \mathrm{e}^{-T\left(m^{2}+l^{2}-2 k_{0} \cdot k \Delta\left(u_{0}, u\right)\right),}
\end{aligned}
$$

where we have abbreviated $l_{\mu} \equiv p_{\mu}+u k_{\mu}+u_{0} k_{0 \mu}$. Eq. (18) after some algebraic manipulations simplifies to

$$
\begin{aligned}
\Gamma\left[p, p^{\prime}\right]= & -\kappa e \int_{0}^{\infty} d T \int_{0}^{1} d u d u_{0}\left(-2 T^{2}\right)\left[(\varepsilon \cdot l)-\left(\varepsilon \cdot k_{0} \Delta^{\bullet}\left(u_{0}, u\right)\right)\right] \\
& \times\left[-2(k \epsilon l) \bullet \Delta\left(u_{0}, u\right)+(l \epsilon l)+(k \epsilon k)^{\bullet} \Delta^{2}\left(u_{0}, u\right)\right] \mathrm{e}^{-T\left(m^{2}+l^{2}-2 k_{0} \cdot k \Delta\left(u_{0}, u\right)\right)} \\
& +\kappa e \varepsilon \epsilon\left(p^{\prime}-p\right) \int_{0}^{\infty} d T T \int_{0}^{1} d u \mathrm{e}^{-T\left[m^{2}+p^{2}+2 p \cdot\left(k+k_{0}\right) u+2 k_{0} \cdot k u\right]}
\end{aligned}
$$

The two different orderings $u \geq u_{0}$ or $u_{0} \geq u$ of the parameter integrals correspond to the two different Born diagrams of Fig. 1, and the seagull diagram comes from the delta function contained in $\boldsymbol{\Delta}^{\bullet}$. Performing the parameter integrals and truncating the external scalar propagators, we get for the sum of these three diagrams

$$
\Gamma\left[p, p^{\prime}\right]=-\frac{2 \kappa e}{2 p \cdot k_{0}}\left(\varepsilon \cdot p^{\prime}\right)(p \epsilon p)+\frac{2 \kappa e}{2 p \cdot k}(\varepsilon \cdot p)\left(p^{\prime} \epsilon p^{\prime}\right)+\kappa e \varepsilon \epsilon\left(p^{\prime}-p\right) .
$$

It remains to construct the $\gamma$-pole diagram. To do so, we decompose this diagram as shown in Fig. 2. The left-hand part, containing a coupling of two scalars with a photon, in the world line formalism corresponds to a self-contraction of a single photon vertex operator. The right-hand part, involving the coupling of two photons 
to a graviton, can be obtained by contracting the graviton field $h_{\mu \nu}$ with the energymomentum tensor $T^{\mu \nu}$. The first part leads to

$$
\begin{aligned}
\Gamma_{s s \gamma}=-i e \int_{0}^{\infty} d T \mathrm{e}^{-m^{2} T} \int_{x(0)=x^{\prime}}^{x(T)=x} \mathcal{D} x(\tau) \mathrm{e}^{-\frac{1}{4} \int_{0}^{T} d \tau \dot{x}^{2}} V^{\gamma}\left[k^{\prime}, \varepsilon^{\prime}\right] \\
=-e \varepsilon_{\mu}^{\prime} \int_{0}^{1} d u \int_{0}^{\infty} d T\left[-2 T\left(k^{\prime \mu} u+p^{\mu}\right)\right. \\
\left.\quad+2 T k^{\prime \mu \bullet} \Delta(u, u)\right] \mathrm{e}^{-T\left(m^{2}+\left(k^{\prime} u+p\right)^{2}-k^{\prime 2} \Delta(u, u)\right)} \\
=2 e \int_{0}^{1} d u \int_{0}^{\infty} d T T\left[\varepsilon^{\prime} \cdot\left(p+\frac{1}{2} k^{\prime}\right)\right] \mathrm{e}^{-T\left[m^{2}+p^{2}+\left(2 p \cdot k^{\prime}+k^{\prime 2}\right) u\right]} \\
=-e \int_{0}^{1} d u \int_{0}^{\infty} d T T\left[\varepsilon^{\prime} \cdot\left(p^{\prime}-p\right)\right] \mathrm{e}^{-T\left[m^{2}+p^{2}+\left(2 p \cdot k^{\prime}+k^{\prime 2}\right) u\right]}
\end{aligned}
$$

For the second part, we use

$$
T^{\mu \nu}=F^{\mu \alpha} F_{\alpha}^{\nu}-\frac{1}{4} F_{\alpha \beta} F^{\alpha \beta} \eta^{\mu \nu} .
$$

Specializing the field strength tensor to $F^{\mu \nu}=f^{\mu \nu}+f^{\prime \mu \nu}$, with $f^{\mu \nu} \equiv k^{\mu} \varepsilon^{\nu}-\varepsilon^{\mu} k^{\nu}$ the photon field strength tensor, $h_{\mu \nu} T^{\mu \nu}$ contains a trilinear term

$$
h_{\mu \nu}\left(f^{\mu \alpha} f_{\alpha}^{\prime \nu}+f^{\prime \mu \alpha} f_{\alpha}^{\nu}\right)-\frac{1}{2} h_{\mu}^{\mu} f^{\alpha \beta} f_{\alpha \beta}^{\prime} .
$$

Using the fact that $h_{\mu}^{\mu}=0$, we get

$$
\begin{aligned}
\Gamma_{g \gamma \gamma} & =\frac{\kappa}{2} h_{\mu \nu} T^{\mu \nu} \\
& =-\kappa\left[\left(k \epsilon k^{\prime}\right)\left(\varepsilon \cdot \varepsilon^{\prime}\right)-\left(k \epsilon \varepsilon^{\prime}\right)\left(\varepsilon \cdot k^{\prime}\right)-\left(\varepsilon \epsilon k^{\prime}\right)\left(\varepsilon^{\prime} \cdot k\right)+\left(\varepsilon \epsilon \varepsilon^{\prime}\right)\left(k \cdot k^{\prime}\right)\right] .
\end{aligned}
$$

Now we have everything to calculate the $\gamma$-pole diagram. We sew the two parts together using the photon propagator in Feynman gauge $\left(\varepsilon^{\prime \alpha} \varepsilon^{\prime \beta} \rightarrow \delta^{\alpha \beta} / k^{\prime 2}\right)$ to get

$$
\begin{aligned}
\Gamma\left[p, p^{\prime}\right]_{\gamma-p o l e} & =-\frac{e \kappa}{2 k_{0} \cdot k} \int_{0}^{1} d u \int_{0}^{\infty} d T T e^{-T\left[m^{2}+p^{2}+2 p \cdot\left(k+k_{0}\right) u+2 k_{0} \cdot k u\right]}\left[(k \epsilon k) \varepsilon \cdot\left(p^{\prime}-p\right)\right. \\
& \left.-k \epsilon\left(p^{\prime}-p\right)\left(\varepsilon \cdot k_{0}\right)-(\varepsilon \epsilon k) k \cdot\left(p^{\prime}-p\right)+\varepsilon \epsilon\left(p^{\prime}-p\right)\left(k \cdot k_{0}\right)\right] .
\end{aligned}
$$

After evaluating the $T$ and $u$-integrals and the removal of the external scalar propagators, Eq. (25) leads to

$$
\begin{aligned}
\Gamma\left[p, p^{\prime}\right]_{\gamma-p o l e}=-\frac{e \kappa}{2 k_{0} \cdot k}[ & {\left[(\epsilon \epsilon k) \varepsilon \cdot\left(p^{\prime}-p\right)-k \epsilon\left(p^{\prime}-p\right)\left(\varepsilon \cdot k_{0}\right)\right.} \\
& \left.-(\varepsilon \epsilon k) k \cdot\left(p^{\prime}-p\right)+\varepsilon \epsilon\left(p^{\prime}-p\right)\left(k \cdot k_{0}\right)\right] .
\end{aligned}
$$

The final result for this scattering amplitude is the sum of Eq. (20) and Eq. (26):

$$
\begin{aligned}
\Gamma\left[p, p^{\prime}\right]_{\gamma s \rightarrow g s} & =\Gamma\left[p^{\prime}, p\right]+\Gamma\left[p^{\prime}, p\right]_{\gamma-p o l e} \\
& =-\frac{\kappa e}{p^{\prime} \cdot k}\left(\varepsilon \cdot p^{\prime}\right)(p \epsilon p)+\frac{\kappa e}{p^{\prime} \cdot k_{0}}(\varepsilon \cdot p)\left(p^{\prime} \epsilon p^{\prime}\right)+\kappa e \varepsilon \epsilon\left(p^{\prime}-p\right)
\end{aligned}
$$


N. Ahmadiniaz et al.

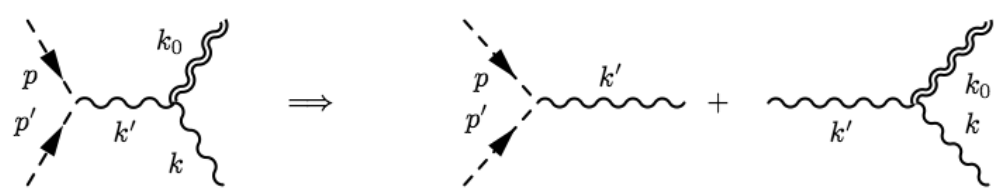

Fig. 2. Decomposition of the $\gamma$-pole diagram into the $s s \gamma$ coupling and the $\gamma \gamma g$ coupling.

$$
\begin{aligned}
-\frac{e \kappa}{2 k_{0} \cdot k}[ & (k \epsilon k) \varepsilon \cdot\left(p^{\prime}-p\right)-k \epsilon\left(p^{\prime}-p\right)\left(\varepsilon \cdot k_{0}\right) \\
& \left.-(\varepsilon \epsilon k) k \cdot\left(p^{\prime}-p\right)+\varepsilon \epsilon\left(p^{\prime}-p\right)\left(k \cdot k_{0}\right)\right] .
\end{aligned}
$$

Now we can compare our final result with the findings of Ref. 2. For graviton photoproduction, $\gamma+s \rightarrow g+s$, Eq. (27) is in agreement with their results.

\section{Factorization Property}

This amplitude is known to have the following factorization property. ${ }^{1-3}$ After factorizing the graviton polarization tensor as $\epsilon_{\mu \nu}=\epsilon_{\mu} \epsilon_{\nu}$, one can also factorize the whole amplitude as

$$
\Gamma\left[p, p^{\prime}\right]_{\gamma s \rightarrow g s}=H \Gamma_{\text {Compton }}(\gamma s \rightarrow \gamma s),
$$

where

$$
\Gamma_{\text {Compton }}(\gamma s \rightarrow \gamma s)=-2 e^{2}\left(\varepsilon \cdot \epsilon+\frac{\varepsilon \cdot p \epsilon \cdot p^{\prime}}{p \cdot k_{0}}+\frac{\varepsilon \cdot p^{\prime} \epsilon \cdot p}{p^{\prime} \cdot k}\right)
$$

is the scalar QED Compton scattering amplitude, and $^{3}$

$$
H=\frac{\kappa}{2 e} \frac{\left(\epsilon \cdot p^{\prime}\right)\left(k_{0} \cdot p\right)-(\epsilon \cdot p)\left(k_{0} \cdot p^{\prime}\right)}{k \cdot k_{0}} .
$$

The way this factorization arises in our formalism is that, if we first consider the Born and seagull diagrams, the structure in Eq. (28) already appears, albeit with some extra terms. Those extra terms are then cancelled by the $\gamma$-pole diagram contributions. We hope that this observation eventually will lead to the establishment of some rules for finding the factorization properties of multi-graviton amplitudes without actually having to compute diagrams of the $\gamma$-pole type.

\section{Conclusion}

In this contribution we presented our recent study of the Compton scattering amplitude for the process $\gamma+s \rightarrow g+s$ or its inverse. From the worldline action we get the Born and seagull diagrams, but the $\gamma$-pole diagram, implied by general covariance, has been treated in a mixed worldline-Feynman approach. For generic tree-level expressions involving an arbitrary number of external photons and gravitons - but no pole-diagrams - a Bern-Kosower type ${ }^{13}$ of master formula can be obtained. ${ }^{14}$ Interestingly the integrand of this formula is expressed in terms of wordline Poincarè 
invariant Green's functions, akin to other results recently obtained for scalar QED ${ }^{4}$ and QCD. ${ }^{5}$ The (photon-irreducible part of the) gravitational Compton scattering, discussed in the present manuscript, is a special case of that master formula.

\section{Acknowledgments}

We would like to thank F. Bastianelli and S. P. Kim for helpful discussion. C. Schubert thanks CONACYT for financial support.

\section{References}

1. S. Y. Choi, J. S. Shim and H. S. Song, Phys. Rev. D 48, 769 (1993); Phys. Rev. D 51, 2751 (1995).

2. B. R. Holstein, Phys. Rev. D 74, 085002 (2006); Am. J. Phys. 74, 1002 (2006).

3. N.E.J. Bjerrum-Bohr, B. R. Holstein, L. Planté and P. Vanhove, Phys. Rev D 91, 064008 (2015), arXiv: gr-qc/1410.4148.

4. N. Ahmadiniaz, A. Bashir and C. Schubert, Phys. Rev. D 93, 045023 (2016), arXiv: hep-ph/1511.05087.

5. N. Ahmadiniaz, F. Bastianelli and O. Corradini, Phys. Rev. D 93, 025035 (2016), arXiv: hep-th/1508.05144.

6. R. P. Feynman, Phys. Rev. 80, 440 (1950); Phys. Rev. 84, 108 (1951).

7. C. Schubert, Phys. Rept. 355, 73 (2001), arXiv: hep-th/0101036.

8. F. Bastianelli and P. van Nieuwenhuizen, Path integrals and anomalies in curved space, Cambridge University press, 2006.

9. N. Ahmadiniaz, F. Bastianelli, O. Corradini, J. M. Davila and C. Schubert, AIP Conf. Proc., 1548221 (2013).

10. F. Bastianelli, O. Corradini, J. M. Dávila and C. Schubert, Phys. Lett. B 716, 345 (2012), arXiv: hep-th/1202.4502.

11. F. Bastianelli, J. M. Dávila and C. Schubert, JHEP 0903, 086 (2009), arXiv: hepth/0812.4849.

12. F. Bastianelli and C. Schubert, JHEP 0502, 069 (2005), arXiv: gr-qc/0412095.

13. Z. Bern, D. Kosower, Phys. Rev. Lett. 66, 1669 (1991); Nucl. Phys. B 362, 389 (1991).

14. N. Ahmadiniaz, O. Corradini, J. M. Dávila and C. Schubert, in preparation. 